\title{
Analysis of the Level of Dysphagia, Anxiety, and Nutritional Status Before and After Speech Therapy in Patients with Stroke
}

\author{
Daniela Drozdz ${ }^{1}$ Renata Mancopes ${ }^{2}$ Ana Maria Toniolo Silva ${ }^{3}$ Caroline Reppold ${ }^{4}$
}

${ }^{1}$ MSc, Human Communication Disorder, Universidade Federal de Santa Maria, Santa Maria, RS, Brazil

2 PhD, Linguistics, Universidade Federal de Santa Catarina, Florianópolis, SC, Brazil

3 PhD, Human Communication Disorder, Universidade Federal de São Paulo, São Paulo, SP, Brazil

${ }^{4}$ PhD, Universidade São Francisco, São Paulo, SP, Brazil

Int Arch Otorhinolaryngol 2014;18:172-177.

\author{
Address for correspondence Daniela Drozdz, MSc, Pós Graduação em \\ Distúrbios da Comunicação Humana, UFSM, Av Fernando Ferrari, 1220 \\ ap 302 Av Fernando Ferrari, 1220 Santa Maria, RS 97050801, Brazil \\ (e-mail: danidrozdz@hotmail.com).
}

\begin{abstract}
Introduction: The rehabilitation in oropharyngeal dysphagia evidence-based implies the relationship between the interventions and their results.

Objective: Analyze level of dysphagia, oral ingestion, anxiety levels and nutritional status of patients with stroke diagnosis, before and after speech therapy.

Method: Clinical assessment of dysphagia partially using the Protocol of Risk Assessment for Dysphagia (PARD), applying the scale Functional Oral Intake Scale for Dysphagia in Stroke Patients (FOIS), Beck Anxiety Inventory (BAI) and the Mini Nutritional Assessment $M N A^{\circledR}$. The sample consisted of 12 patients, mean age of 64.6 years, with a medical diagnosis of hemorrhagic and ischemic stroke and without cognitive disorders. All tests were applied before and after speech therapy ( 15 sessions). Statistical analysis was performed using the chi-square test or Fisher's exact test, McNemar's test, Bowker's symmetry test and Wilcoxon's test.

Results: During the pre-speech therapy assessments, $33.3 \%$ of patients had mild to moderate dysphagia, $88.2 \%$ did not receive food orally, $47.1 \%$ of the patients showed malnutrition and $35.3 \%$ of patients had mild anxiety level. After the therapy sessions, it was found that $33.3 \%$ of patients had mild dysphagia, $16.7 \%$ were malnourished and $50 \%$ of patients had minimal level of anxiety.

\section{Keywords}

- swallowing disorders

- anxiety

- nutritional status

- rehabilitation

Conclusion: There were statistically significant evolution of the level of dysphagia $(p=0.017)$ and oral intake $(p=0.003)$ post-speech therapy. Although not statistically significant, there was considerable progress in relation to the level of anxiety and nutritional status.
\end{abstract}

\section{Introduction}

Swallowing is a complex act that requires control and regulation at all levels of the nervous system. Changes in this process, such as incoordination or weakness of the biomechanics of this function, characterize dysphagia. ${ }^{1}$

received

October 16, 2013

accepted

November 18, 2013
DOI http://dx.doi.org/

10.1055/s-0033-1364169. ISSN $1809-9777$.
One study found that between 42 and $67 \%$ of patients who are affected by stroke have dysphagia, making it the most common result in these cases. The diagnosis of oropharyngeal dysphagia is associated with laryngotracheal aspiration, which may result in pulmonary diseases, malnutrition, dehydration, and death. ${ }^{2-5}$ Among the already recognized consequences of

Copyright $(2014$ by Thieme Publicações License terms Ltda, Rio de Janeiro, Brazil
(ब(1) $\Theta$ 
dysphagia, it is also necessary to note the possibility of psychological disorders related to emotional modifications that may generate changes in the swallowing process. ${ }^{6}$

With the improvement in the care and treatment of stroke, it is important that therapeutic work should not only be focused on the acute phase, because the next or chronic phase can last indefinitely, and its sequelae, such as oropharyngeal dysphagia, should receive the same attention. The treatment during the chronic phase aims to improve the performance of functional tasks and reintegrate the patient to their daily activities. ${ }^{7}$

It is currently necessary to identify, classify, and organize the goals and procedures of rehabilitation in the care of patients with oropharyngeal dysphagia, which would improve the speech therapy in the rehabilitation process. ${ }^{8}$

A recent study found that the literature lacks reports of the indicators resulting from dysphagia speech therapy in the hospital. The use of functionality scales, besides the possibility to manage the results of this study, allows comparison of results before and after speech therapy to analyze the effectiveness of treatment and possible actions to be improved. ${ }^{8}$

The aim of this study was to analyze the level of dysphagia, oral ingestion, anxiety, and nutritional status before and after speech therapy in the Hospital Universitário de Santa Maria (HUSM) in patients with stroke.

\section{Methods}

This quantitative cross-sectional study was conducted by collecting data of adult patients of both sexes admitted to the HUSM with neurogenic oropharyngeal dysphagia. This study was approved by the Ethics in Research Committee of the institution of origin under number 0196.0.243.000-11.

Inclusion criteria specified adult patients of both sexes with neurogenic oropharyngeal dysphagia, stroke diagnosis, stable condition for the realization of the screening, plans to be admitted to hospital, and clinical signs of aspiration and/or complaints of dysphagia.

Exclusion criteria specified having previously undergone speech therapy and having a diagnosis of mechanical dysphagia and craniofacial malformations.

All patients were in acute phase and were evaluated about 8 days after admission. Initially, clinical assessment of swallowing in bed was performed by using partial Protocol of Risk Assessment for Dysphagia ${ }^{9}$ (testing only volumes of 3, 5, 10 and $20 \mathrm{~mL}$ ) for the determination and classification of the diagnosis of oropharyngeal dysphagia. In this assessment, the patient sat upright in bed (90 degrees). Swallowing of water and pasty food was observed only in volumes of 3 and $5 \mathrm{~mL}$. Larger volumes (10 and $20 \mathrm{~mL}$ ) were used when necessary to facilitate the identification of signs suggestive of penetration/ aspiration and defining the conduct. ${ }^{10}$ The water was supplied with a glass, and the pasty food was offered with a spoon.

The general appearance of the patient was analyzed, along with symmetry and posture and mobility and tone of the organs and muscles involved in chewing and swallowing (lips, teeth, jaw, palatoglossus muscle, palatopharyngeal muscle, and the posterior pharyngeal wall). The extra- and intraoral sensitivity was also observed. Presence or absence of gagging or coughing during and after swallowing, the labial sealing, the movement of the tongue, and the use of compensatory movements to propel the food bolus were observed. During the assessment, cervical auscultation was used to verify the presence or absence of noise before or after swallowing. Furthermore, as defined in the protocol, the oximeter was used to check the heart rate and oxygen saturation during swallowing. Then, the Functional Oral Intake Scale (FOIS) ${ }^{11}$ was used to characterize the level of the patient's oral intake.

To assess the level of anxiety of the patient or family/ caregiver, the Beck Anxiety Inventory (BAI) was applied using a version translated and validated by Cunha in $2011 .^{12}$ The Beck Anxiety Inventory consists of 21 items related to signs and symptoms of anxiety, with responses ranging from none, slight, moderate, and severe, graded 0 to 3, respectively. The recommended rating for the level of anxiety is minimal ( 0 to 7$)$, mild ( 8 to 15 ), moderate (16 to 25 ), and severe (26 to 63 ).

The nutritional status was assessed by means of the Mini Nutritional Assessment (MNA). ${ }^{13}$ This screening aims to investigate changes in food intake, weight loss, mobility, psychological stress, neurologic problems, and body mass index. The maximum score is 14 points, classifying the patient's nutritional status as normal (12 to 14 points), at risk of malnutrition (8 to 11 points), or malnourished (0 to 7 points).

After initial assessments and confirming the degree of oropharyngeal dysphagia in different levels, the speech therapy began in the bed to restore the oral and/or weaning of alternative routes of supply, according to the specific need of each case. Patients were treated for 15 sessions of 40 minutes each, according to a previous study. ${ }^{14}$ They were assessed about 8 days after admission and treated in the hospital bed. The goal of speech therapy is to promote a progressive decrease in enteral supply, safely aiding the progression of oral feeding whenever possible. This approach used direct and indirect therapies. The indirect therapy aims to improve the physiology of swallowing. The exercises used for this purpose were isotonic muscle contraction and cryostimulation associated with sour taste. In direct therapy, compensation strategies were used (cleaning maneuvers and Masako's maneuvers), encompassing the external control of the swallowing process. Through that, the purpose was to preserve food quality and safety and to maintain adequate nutritional status. $^{6,15-17}$

The procedures performed during this study (data collection and therapeutic treatment) were made by the researcher speech therapist and also by residents of the college hospital staff. It is noteworthy that the entire therapeutic process was outlined by the researcher speech therapist in charge.

To describe the profile of the sample according to the variables under study, frequency tables of the categorical variables were made, with values of absolute frequency $(n)$ and percentage (\%) and descriptive statistics of the numeric variable with mean values, standard deviation, minimum and maximum values, medians, and quartiles.

To examine the relationship between the categorical variables in each moment, the chi-square test or the Fisher exact test was used in the presence of expected values below five. To compare the categorical variables between assessments 
before and after therapy, we used the McNemar test for two categories and the Bowker symmetry test for three or more categories. To compare the ordinal categorical variables between the ratings before and after therapy, the Wilcoxon test was used for related samples. The significance level for the statistical tests was $5 \%$ (i.e., $p<0.05$ ).

\section{Results}

Eighteen patients (14 men and 4 women), age between 38 and 85 years (mean 64.6 years), participated in this study; all had the first episode of stroke certified by medical diagnosis in the records, which were determined as hemorrhagic (10 patients) or ischemic (8 patients). Patients did not have cognitive disorders. All patients were in the acute phase of the disease. They were assessed about 8 days after admission and treated in the hospital bed. Of these, two died, one was in a coma, two were transferred to the city hospital of origin before starting speech therapy, and one was discharged by the doctor before starting the assessments. Therefore, the sample consisted of 12 patients. The average length of stay of these patients was 30 days. The profiles of the patients according to the degree of dysphagia, level of oral intake, nutritional status, and level of anxiety before and after speech therapy are described in - Tables 1-4.

- Table 5 shows the comparative analysis of the numerical variables before and after speech therapy. - Table 6 shows the comparison before and after speech therapy between the variables degree of dysphagia, anxiety inventory, and nutritional assessment.

During this research, it was observed through pre-speech therapy clinical assessment that $33.3 \%$ of patients had mild to moderate dysphagia and $25 \%$ of patients had mild dysphagia. Functional swallowing was found in $16.7 \%$ of patients. After 15 therapeutic treatment sessions, $33.3 \%$ of patients had mild dysphagia and $8.3 \%$ had severe dysphagia in the clinical assessment. The diagnosis of severe dysphagia after the therapy sessions were due to the worsening of the neurologic status of the patient under care ( - Table $\mathbf{1}$ ).

- Table 2 shows that the number of patients not receiving oral feeding before therapy decreased after speech therapy. It

Table 1 Descriptive analysis of degree of dysphagia

\begin{tabular}{|l|l|l|}
\hline \multirow{2}{*}{ Classification of the dysphagia } & \multicolumn{2}{|c|}{ Clinical assessment $^{\mathrm{a}}$} \\
\cline { 2 - 3 } & Before (\%) & After (\%) \\
\hline Normal & 0 & 16.7 \\
\hline Functional swallowing & 16.7 & 25.0 \\
\hline Mild dysphagia & 25.0 & 33.3 \\
\hline Mild to moderate dysphagia & 33.3 & 16.7 \\
\hline Moderate dysphagia & 0 & 0 \\
\hline Moderate to severe dysphagia & 25.0 & 0 \\
\hline Severe dysphagia & 0 & 8.3 \\
\hline Total & 100 & 100 \\
\hline
\end{tabular}

${ }^{a}$ Classification according to Padovani et al. ${ }^{9}$
Table 2 Descriptive analysis of the level of oral intake

\begin{tabular}{|l|l|l|}
\hline \multirow{2}{*}{ Functional Oral Intake Scale $^{\mathbf{a}}$} & \multicolumn{2}{|c|}{} \\
\cline { 2 - 3 } & Before (\%) & After (\%) \\
\hline 1 & 88.2 & 8.3 \\
\hline 2 & 11.8 & 0 \\
\hline 3 & 0 & 16.7 \\
\hline 4 & 0 & 8.3 \\
\hline 5 & 0 & 25.0 \\
\hline 6 & 0 & 0 \\
\hline 7 & 0 & 41.7 \\
\hline Total & 100 & 100 \\
\hline
\end{tabular}

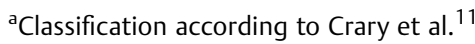

was also observed after the treatment that more than $40 \%$ of patients had a total feed orally released.

During application of the nutritional screening through the MNA, before speech therapy $47.1 \%$ of patients had malnutrition, $41.2 \%$ were at nutritional risk, and $11.7 \%$ had normal nutritional status. After the therapy sessions, $16.7 \%$ were malnourished, $66.7 \%$ were at risk of malnutrition, and $16.7 \%$ were in normal nutritional status ( - Table 3 ).

When analyzing the results of pretherapy BAI, ${ }^{12} 35.3 \%$ of patients had a mild degree of anxiety and $11.8 \%$ had a severe degree of anxiety, and after speech therapy, $50 \%$ of patients had minimal degree of anxiety and $8.3 \%$, a moderate degree (-Table 4).

In this research, the degree of dysphagia established by the clinical assessment ${ }^{9}$ and the FOIS scale of oral ingestion ${ }^{11}$ significantly improved after the speech therapy (- Table $\mathbf{5}$ ). These results illustrate that the therapy promoted the evolution of the oral intake, with consequent changes in their general status. $^{18}$

- Table 6 shows the comparison before and after speech therapy between degree of dysphagia, anxiety inventory, and nutritional assessment. No significant results were found in this comparison.

\section{Discussion}

Rehabilitation demonstrates the importance of interventions and their results. Adding to this, early diagnosis and targeted treatment can reduce both the complications related to

Table 3 Descriptive analysis of the nutritional status

\begin{tabular}{|l|l|l|}
\hline \multirow{2}{*}{ Mini Nutritional Assessment } & \multicolumn{2}{|c|}{} \\
\cline { 2 - 3 } & Before (\%) & After (\%) \\
\hline Normal & 11.7 & 16.7 \\
\hline At risk of malnutrition & 41.2 & 66.7 \\
\hline Malnourished & 47.1 & 16.7 \\
\hline Total & 100 & 100 \\
\hline
\end{tabular}


Table 4 Descriptive analysis of the level of anxiety

\begin{tabular}{|l|l|l|}
\hline \multirow{2}{*}{ Beck Anxiety Inventory $^{\mathbf{a}}$} & \multicolumn{2}{|c|}{} \\
\cline { 2 - 3 } & Before (\%) & After (\%) \\
\hline Minimum & 29.4 & 50.0 \\
\hline Mild & 35.3 & 41.7 \\
\hline Moderate & 23.5 & 8.3 \\
\hline Severe & 11.8 & 0 \\
\hline Total & 100 & 100 \\
\hline
\end{tabular}

${ }^{a}$ Classification according to Cunha. ${ }^{12}$

dysphagia as well as length of hospital stay, which consequently results in reduced costs. ${ }^{8}$

Stroke is a neurologic disease with higher mortality and sequelae in adults. Studies show a significant incidence of oropharyngeal dysphagia in patients with this neurologic condition, which increases morbidity. The risk of aspiration is also present, and as a result, there can be inadequate oral nutrition. ${ }^{19}$

Research and clinical evidence suggest that by establishing an organized plan of care (screening, diagnosis, and treatment of dysphagia), a significant reduction of consequences can be obtained in the patient with dysphagia. Furthermore, to characterize the effects of therapy using data obtained before and after treatment meets the growing interest in evidence-based practice. Thus, to manage and systematize the routine care of speech therapy can ensure better efficiency. ${ }^{20,21}$

Chronic stroke condition can change individuals' daily living habits and thus bring about a drastic change in their lifestyle. ${ }^{22}$ The duration of the condition may influence the performance of their daily activities, taking into account that better results in rehabilitation are obtained during the first 6 months of onset. ${ }^{23}$

One study reported that dysphagia is present in 42 to $67 \%$ of patients with stroke in the acute phase, and $20 \%$ of patients with this disease will die within the first year, $35 \%$ due to pneumonia occurring during the hospitalization. Pneumonia is related to the presence of aspiration, which maybe be caused by various degrees of dysphagia. ${ }^{2}$ A week after the onset of the disease, $27 \%$ of patients still had the disease;
Table 6 Comparative analysis of the numerical variables dysphagia (Beck Anxiety Inventory), dysphagia (MNA), Beck Anxiety Inventory (MNA) before and after speech therapy

\begin{tabular}{|l|l|l|}
\hline \multirow{2}{*}{ Variables } & \multicolumn{2}{|c|}{$p$ value $^{\mathrm{a}}$} \\
\cline { 2 - 3 } & Before & After \\
\hline Dysphagia (Beck Anxiety Inventory) & 0.895 & 0.349 \\
\hline Dysphagia (MNA) & 0.529 & 0.879 \\
\hline Beck Anxiety Inventory (MNA) & 0.877 & $>0.999$ \\
\hline
\end{tabular}

Abbreviation: MNA, Mini Nutritional Assessment.

a Fisher exact test of related samples for comparison between before and after therapies.

moreover, after 6 months, $8 \%$ of patients demonstrated oropharyngeal dysphagia. ${ }^{24}$

The data in - Table 1 show an increase in the number of patients with improved diagnosis on clinical assessment. The presence of functional swallowing (33.3\%) increased after the sessions of speech therapy, and the percentage of diagnoses of moderate to severe dysphagia and severe dysphagia fell.

A recent study of more than 3,000 patients with heterogeneous disorders (Parkinson disease, stroke, head and neck surgery, dementia, and cardiothoracic surgery) investigated the benefits of water-swallowing test to determine the presence or absence of aspiration and possible procedures for oral feeding. The authors concluded that although the clinical assessment was effective, it should not be the only one for establishing the diagnosis and prognosis of patients with dysphagia, confirming the need for an instrumental accompaniment (videofluoroscopy VFS) in the assessment when the patient shows signs of difficulties to feed upon clinical assessment. ${ }^{25}$

After 15 therapeutic sessions ( - Table 2 ), $41.7 \%$ of patients achieved level 7 of the FOIS oral intake scale. ${ }^{11}$ Safe and effective removal of the alternative pathway of food and/or reintroduction of oral feeding makes possible the assessment of the results of speech therapy in dysphagia. ${ }^{8}$

The percentage of malnourished patients decreased after treatment ( - Table 3 ), demonstrating that the increased oral intake improves the nutritional status of patients with dysphagia. According to Silva et al, ${ }^{26}$ the nutritional status of the patients is occasionally referred to in the literature as a risk characteristic of dysphagia; however, it becomes crucial to observe the degree of impairment of the neurogenic

Table 5 Comparative analysis of level of dysphagia, level of oral intake, and MNA and Beck Anxiety Inventory before and after speech therapy

\begin{tabular}{|l|l|l|l|l|l|l|}
\hline \multirow{2}{*}{ Variable } & \multirow{2}{*}{$\boldsymbol{n}$} & \multicolumn{2}{|c|}{ Average } & \multicolumn{2}{c|}{ SD } & \multirow{2}{*}{$p$ value $^{\mathrm{a}}$} \\
\cline { 3 - 6 } & & Before & After & Before & After & \\
\hline Level (dysphagia) after speech therapy & 12 & 3.92 & 2.75 & 1.44 & 1.22 & $0.017^{*}$ \\
\hline Level (FOIS) & $12^{*}$ & $1.08^{*}$ & $5.08^{*}$ & $0.29^{*}$ & $2.02^{*}$ & $0.003^{*}$ \\
\hline Nutritional status (MNA) & 12 & 2.17 & 2.00 & 0.72 & 0.60 & 0.727 \\
\hline Level (Beck Anxiety Inventory) & 12 & 2.25 & 1.58 & 0.97 & 0.67 & 0.063 \\
\hline
\end{tabular}

Abbreviations: *, statistical significance; SD, standard deviation; FOIS, Functional Oral Intake Scale ; MNA, Mini Nutritional Assessment.

${ }^{a}$ Wilcoxon test of related samples for comparison between before and after therapies. 
dysphagia, considering that even in the absence of laryngotracheal penetration/aspiration, the nutrition state may be compromised. Thus, the authors report that during speech therapy in this population, both lungs should be analyzed.

The indication of food restriction or feeding alternative in patients with dysphagia worsens the nutritional condition, leading to malnutrition. The malnourished patient's ability to swallow is impaired due to changes in muscle and nerve disorders; in addition, malnutrition is an established risk for the patient to develop lung diseases. ${ }^{27}$

According to a study by Crary, ${ }^{28}$ early identification of dysphagia and malnutrition is of great importance. Both were prevalent and were related to unfavorable outcomes in the study sample mentioned. The authors concluded that interventions that aim to improve the ability to swallow may benefit the nutritional status of stroke patients.

The degree of anxiety considered serious was extinguished in the sample and the minimum degree showed an increase to $50 \%$ in the assessment after speech therapy (-Table 4). A survey that assessed symptoms of depression after stroke in 206 patients, also using scales, observed that the loss of appetite (characterized as a somatic symptom in this study) had the second highest score in this population. The authors concluded that, first, depression is the most common symptom in patients suffering from stroke. However, other somatic symptoms should not be overlooked because of their importance in quality of life. ${ }^{29}$

Studies revealed that the presence of dysphagia is associated with anxiety, bringing the individual psychosocial consequences such as depression, fear, and frustration, which can significantly affect quality of life. ${ }^{27,30,31}$ A recent study assessed patients with dysphagia after stroke using the BAI before and after therapy and observed that at the end of the therapy, there was a significant decrease in scores on this scale. $^{32}$ Therefore, the decrease in anxiety found in this sample indicates that, although not statistically significant, developments in the diagnosis of dysphagia promoted a positive evolution in the condition of anxiety.

A survey showed that, upon receiving the diagnosis of dysphagia, patients report a decrease in their quality of life because from that moment meals become a problem and not pleasure. $^{33}$ The increased oral ingestion in this sample promoted a consequent evolution of anxiety, as patients were able to feed normally. Thus, it is possible to infer that the state of dysphagia affects the anxiety state of the individual, and it is necessary to consider this aspect in the process of rehabilitation of oropharyngeal dysphagia.

The change of degree of dysphagia established through clinical evaluation ${ }^{9}$ and the use of FOIS ${ }^{11}$ was statistically significant, indicating evolution in the patients' condition after speech therapy (-Table $\mathbf{5}$ ). These findings confirm the shift in the dynamics of swallowing and subsequent efficacy of the therapeutic program. The application of this type of procedure before and after therapy for clinical assessment is important to assess the best treatment for each case and to analyze objectively the evolution of each patient. ${ }^{34}$

Despite the nutritional scale and the Beck inventory not showing statistically significant results, a trend toward im- provement could be seen. The change in this sample may be due to the evolution of the diagnosis of dysphagia in these patients, given that, with the improvement of dysphagia, the reestablishment of the general health and quality of life of patients consequently occurs. ${ }^{16}$

The association among dysphagia and the degree of anxiety, dysphagia, and nutritional status and anxiety (- Table 6) was not statistically significant. However, in the isolated analysis of these parameters, one can observe progress after therapy. The small sample size may have affected these results.

Due to the high incidence and prevalence of the diagnosis of oropharyngeal dysphagia in the hospital, it is necessary to use a management program with defined goals and objectives. Adapting protocols and therapeutic processes becomes essential for the work of the speech therapist in dysphagia to be more efficient and effective, facilitating the identification of the population served and improving the procedures to which they will be offered. ${ }^{8,35}$

\section{Conclusion}

The degree of dysphagia and the oral intake level showed significant differences before and after speech therapy. Although not statistically significant, there was considerable progress in relation to the level of anxiety and nutritional status. Studies with larger samples are suggested.

\section{References}

1 Mangili LD, Moraes DP, Medeiros C. Protocolo de avaliação fonoaudiológica preliminar (PAP). DISFAGIA-Prática Baseada em Evidências. São Paulo, Brazil: Sarvier; 2012:45-61

2 Hinchey JA, Shephard T, Furie K, Smith D, Wang D, Tonn S; Stroke Practice Improvement Network Investigators. Formal dysphagia screening protocols prevent pneumonia. Stroke 2005;36(9): 1972-1976

3 Foley N, Teasell R, Salter K, Kruger E, Martino R. Dysphagia treatment post stroke: a systematic review of randomised controlled trials. Age Ageing 2008;37(3):258-264

4 Padovani AR. Protocolo fonoaudiológico de introdução e transição da alimentação por via oral para pacientes com risco para disfagia (PITA) [94 f. Dissertação (Mestrado em Ciências)]. São Paulo, Brazil: Faculdade de Medicina da Universidade de São Paulo; 2010

5 Andrade CRF. Prática Baseada em Evidência na Disfagia. DISFAGIAPrática Baseada em Evidências. São Paulo, Brazil: Sarvier; 2012:3-5

6 Canongia MB, Alves CMM. Disfagia-Estudo e Reabilitação. Rio de Janeiro, Brazil: Revinter; 2010

7 Miller EL, Murray L, Richards L, et al; American Heart Association Council on Cardiovascular Nursing and the Stroke Council. Comprehensive overview of nursing and interdisciplinary rehabilitation care of the stroke patient: a scientific statement from the American Heart Association. Stroke 2010;41(10): 2402-2448

8 Moraes DP, Andrade CRF. Quality indicators for integrated care of dysphagia in hospital settings. J Soc Bras Fonoaudiol 2011;23(1): 89-94

9 Padovani AR, Moraes DP, Mangili LD, et al. Protocolo Fonoaudiológico de Avaliação do Risco para Disfagia (PARD). Rev Soc Bras Fonoaudiol 2007;12(3):199-205 
10 VA. R. G. Disfagia orofaríngea pós-acidente vascular encefálico. Tratado de Fonoaudiologia. São Paulo, Brazil: Roca; 2004:354-369

11 Crary MA, Mann GD, Groher ME. Initial psychometric assessment of a functional oral intake scale for dysphagia in stroke patients. Arch Phys Med Rehabil 2005;86(8):1516-1520

12 Cunha J. Escalas de Beck-Manual. São Paulo, Brazil: Casa do Psicólogo; 2011

13 Vellas B, Villars H, Abellan G, et al. Overview of the MNA-Its history and challenges. J Nutr Health Aging 2006;10(6):456-463, discussion 463-465

14 Silva RG, Jorge AG, Peres FM, Cola PC, Gatto AR, Spadotto AA. Protocolo para controle de eficácia terapêutica em disfagia orofaríngea neurogênica (PROCEDON). Rev CEFAC 2010;12(1):75-81

15 Zaffari RT. Disfagia Orofaríngea Neurogênica-Orientações para cuidadores e familiares. Disfagia-Avaliação e Tratamento. Rio de Janeiro, Brazil: Revinter; 2003:197-208

16 Furkim AM, Mattana AV. Disfagias Neurogênicas: Terapia. Distúrbios neurológicos adquiridos: fala e deglutição. Barueri-SP, Brazil: Manole; 2010:302-319

17 Moraes DP, Alves CF. Programa Fonoaudiológico de Reabilitação da Deglutição em Ambulatório. Disfagia-Prática Baseada em Evidências. São Paulo, Brazil: Sarvier; 2012:86-104

18 Speyer R, Baijens L, Heijnen M, Zwijnenberg I. Effects of therapy in oropharyngeal dysphagia by speech and language therapists: a systematic review. Dysphagia 2010;25(1):40-65

19 Bordon A, Bokhari R, Sperry J, Testa D IV, Feinstein A, Ghaemmaghami V. Swallowing dysfunction after prolonged intubation: analysis of risk factors in trauma patients. Am J Surg 2011; 202(6):679-682, discussion 682-683

20 Baijens LWJ, Speyer R. Effects of therapy for dysphagia in Parkinson's disease: systematic review. Dysphagia 2009;24(1):91-102

21 Andrade CRF, Limongi SCO. Disfagia-Prática baseada em evidências. São Paulo, Brazil: Sarvier; 2012

22 Medeiros MSM. Treinamento de Força em Sujeitos Portadores de Acidente Vascular Cerebral. Rev Dig Vida Saude 2002;1(3):1-21

23 Delboni MCC, Malengo PCM, Schmidt EPR. Relação entre os aspectos das alterações funcionais e seu impacto na qualidade de vida das pessoas com sequelas de Acidente Vascular Encefálico (AVE). O Mundo da Saúde 2010;34(2):165-175

24 Pinto RASR. Neurologia da Deglutição. Disfagias orofaríngeas, vol. 2. Barueri-SP, Brazil: Pro Fono; 2008:1-14

25 Suiter DM, Leder SB. Clinical utility of the 3-ounce water swallow test. Dysphagia 2008;23(3):244-250

26 Silva RG, Motonaga SM, Cola PC, et al. Estudo multicêntrico sobre escalas para grau de comprometimento em disfagia orofaríngea neurogênica. Rev Soc Bras Fonoaudiol. 2012; 17(2):167-170

27 Souza BBA, et al. Nutrição e Disfagia. Curitiba, Brazil: Nutroclínica; 2003

28 Crary MA, Carnaby-Mann GD, Miller L, Antonios N, Silliman S. Dysphagia and nutritional status at the time of hospital admission for ischemic stroke. J Stroke Cerebrovasc Dis 2006;15(4): 164-171

29 de Coster L, Leentjens AFG, Lodder J, Verhey FRJ. The sensitivity of somatic symptoms in post-stroke depression: a discriminant analytic approach. Int J Geriatr Psychiatry 2005;20(4):358-362

30 Ekberg O, Hamdy S, Woisard V, Wuttge-Hannig A, Ortega P. Social and psychological burden of dysphagia: its impact on diagnosis and treatment. Dysphagia 2002;17(2):139-146

31 Eslick GD, Talley NJ. Dysphagia: epidemiology, risk factors and impact on quality of life-a population-based study. Aliment Pharmacol Ther 2008;27(10):971-979

32 Kang JH, Park RY, Lee SJ, Kim JY, Yoon SR, Jung KI. The effect of bedside exercise program on stroke patients with dysphagia. Ann Rehabil Med 2012;36(4):512-520

33 Farri A, Accornero A, Burdese C. Social importance of dysphagia: its impact on diagnosis and therapy. Acta Otorhinolaryngol Ital 2007; 27(2):83-86

34 Santoro PP, Furia CL, Forte AP, et al. Otolaryngology and speech therapy evaluation in the assessment of oropharyngeal dysphagia: a combined protocol proposal. Braz J Otorhinolaryngol 2011; 77(2):201-213

35 Padovani AR, Moraes DP, Sassi FC, Andrade CRF. Clinical swallowing assessment in intensive care unit. CoDAS 2013;25(1):1-7 